\title{
Metachronous multiple carcinomas arising at a colostomy site 18 years after abdominoperineal resection
}

\author{
Norihiro Okamoto
}

Accepted: 3 June 2009 /Published online: 19 June 2009

(C) Springer-Verlag 2009

\section{Dear Editor:}

Metachronous multiple carcinomas at the site of stoma is rare condition after surgery. A median of 264 months interval from the initial operation was documented in 35 cases reported.

In 1958, the following criteria for multiple carcinomas of the colon were defined by Mortel: (1) malignancy of a lesion, as indicated by histopathological examination; (2) synchronous carcinomas, with normal mucosa existing between the tumors; and (3) for metachronous carcinomas, a tumor, except for a tumor occurring at an anastomosis, which is not suspected of being an invasion, metastasis, or recurrence of another tumor. They reported that multiple carcinomas accounted for $4.3 \%$ of all colorectal carcinomas and for $12.9 \%$ of colorectal carcinomas and polypoid carcinoma of the colon. In 1996, the Cancer Registry of the Japanese Society for Cancer of the Colon and Rectum reported that multiple carcinomas occurred in $9.7 \%$ of patients (synchronous, $4.3 \%$; metachronous, $5.4 \%$ ). To our knowledge, 45 cases of stoma carcinomas that include both synchronous and metachronous carcinomas have been reported in Japan. Among them, 35 cases (25 men, median $=68$ years, range $=38-88$ years , including the present case, fulfilled the above criteria for metachronous multiple carcinomas arising at the site of colostomy. Thirty of the 35 cases underwent abdominoperineal resection for rectal carcinoma. The median time of development from initial operation to second tumor was 264 months (range= 19-504 months). In 24 of the 35 cases (68.6\%), the second tumor was discovered more than 10 years after the initial surgery. As for the histology, adenocarcinoma was confirmed in 30 of the 35 cases $(85.7 \%)$ and mucinous adenocarcinoma in three cases. These proportions were equivalent to common advanced cases in our series. In the present case, the patient did not recognize the possibility of carcinoma occurrence or growth more than 10 years. The fact suggests that most of the patients might not recognize the occurrence of a carcinoma in a stoma. Occurrence of carcinoma in the stoma site is a rare condition, but carcinoma can occur in a stoma. The refined observation of a stoma might be suggested for the ostomate for long period after stoma creation.
N. Okamoto $(\bowtie)$

Department of Colorectal Surgery, Shinshiro Municipal Hospital, 32-1, Kitahata,

Shinshiro, Aichi 441-1387, Japan

e-mail: norihiro_okamoto@yahoo.co.jp 